\title{
Is Molecular Diagnosis Necessary for Children with Duchenne Muscular Dystrophy?
}

\author{
Ratna Dua Puri \\ From the Institute of Medical Genetics and Genomics, Sir Ganga Ram Hospital, New Delhi, India \\ ratnadpuri@yahoo.com
}

$\mathrm{T}$ The importance of Duchenne muscular dystrophy (DMD) for the pediatrician is in the fact that it is the commonest muscular dystrophy presenting in childhood with an incidence of 1 in 3500 live male births, and also due to the emergence of exciting novel therapies in recent years. Mutations in the $D M D$ gene that codes for the dystrophin protein are associated with the severe phenotype of X-linked progressive muscular dystrophy, DMD, or the milder Becker muscular dystrophy (BMD). Traditionally, diagnosis of DMD was established by clinical evaluation with or without enzyme immunehistochemistry for dystrophin.

The $D M D$ gene is the largest gene in humans spanning 79 exons, and predominantly expresses in the skeletal and cardiac muscle with a small amount in the brain [1]. In this issue of Indian Pediatrics, Tallapaka, et al. [2] characterize the mutation spectrum in patients presenting with a DMD phenotype, and demonstrate the decreasing role of invasive muscle biopsy with increasing availability of non-invasive molecular testing. International comprehensive guidelines for the management and care of patients with DMD for practitioners recommend identification of the mutation in patients. Molecular testing is important for confirming the diagnosis amidst a plethora of phenotypically similar muscular dystrophies with high creatine kinase (CK), for counseling families about recurrence risk and prenatal diagnosis, and for carrier detection of at-risk family members. In our experience, there exist challenges in counseling for Xlinked disorders in India due to the predominantly patriarchal society. Hence, utmost care is needed while counseling at-risk carrier females. Though, carrier females are mostly asymptomatic, monitoring for cardiac complications and management as needed is important.

Mutations in the gene are predominantly deletions $(60-70 \%)$ or duplications $(5-10 \%)$, concentrated in two hot spot regions between exons 45-55 and 2-10 for deletions and duplications, respectively [3-5]. Tallapaka, et al. [3] in their study, also found $73 \%$ of mutations concentrated in these two regions. Appropriately, they opined that Multiplex Ligation-dependent Probe Amplification (MLPA) remains the first line investigation for diagnosis of DMD as it tests for deletions and duplications in all 79 exons of the gene compared to multiplex Polymerase chain reaction (PCR) that does not test all exons or define the extent of the deletion. Single exon deletions should be confirmed by an alternative technique [6]. As availability no longer remains the issue in India, awareness of the appropriate test is essential. $25 \%-35 \%$ of pathogenic variants in DMD and about $10 \%-20 \%$ of males with BMD have single nucleotide variations, small deletions and insertions or splice site changes identified by sequencing [3]. Given the high cost of Sanger sequencing for this large gene, next generation techniques remain the most cost-effective test currently for this cohort of MLPAnegative patients [4]. In the near future, we can expect a single step test to evaluate all mutations types in DMD gene [6].

The 79 exons of the DMD gene have a high mutation rate, and in one of three cases the phenotype occurs due to a de novo mutation that is not inherited [7]. Thus, new cases will arise despite the best preventive measures in families with a history of DMD.

The utility of a timely molecular diagnosis is multifold. Phenotype based on an inframe deletion or duplication that will not disrupt the reading frame allows translation of the mRNA into a smaller but active protein. As this protein is active, the phenotype is the milder BMD. The severe phenotype of DMD occurs when the deleted/duplicated exons or a premature stop codon impacts translation, and the resulting truncated dystrophin protein is functionally unstable [6].

Corticosteroids are currently the accepted standard of care. The past decade has witnessed many advances in definitive therapies for DMD. Newer personalized 
therapies are targeted to work on patients with specific mutation types [8]. Fig. 1 illustrates what is currently available and approved by the Food and Drugs Administration (FDA) and European Medicines Agency (EMA) for the treatment of DMD, and therapies that are in the pipeline.

The article by Tallapaka, et al. [2] is very timely in this era of personalized medicine. Understanding the mutation spectrum in the population allows development of cost-effective diagnostic protocols as also define the population potentially amenable to the existing/ developing targeted therapies.

Funding: None; Competing interest: None stated.

\section{REFERENCES}

1. Falzarano MS, Scotton C, Passarelli C, Ferlini A. Duchenne muscular dystrophy: From diagnosis to therapy. Molecules. 2015;20:18168-84.

2. Tallapaka K, Ranganath P, Ramachandran A, Uppin MS, Perala S, Aggarwal S, et al. Molecular and histopathological characterization of patients presenting with the Duchenne muscular dystrophy phenotype in a tertiary care center in Southern India. Indian Pediatr. 2019;56:556-9.

3. Darras BT, Urion DK, Ghosh PS. Dystrophinopathies. 2000 Sep 5. In: Adam MP, Ardinger HH, Pagon RA, Wallace SE (editors). GeneReviews ${ }^{\circledR}$. Seattle (WA): University of Washington, Seattle; 1993-2019. Available from: https://www.ncbi.nlm.nih.gov/books/NBK1119/. Accessed June 18, 2019.

4. Birnkrant DJ, Bushby K, Bann CM, Apkon SD, Blackwell A, Brumbaugh D, et al. DMD Care Considerations Working Group. Diagnosis and management of Duchenne muscular dystrophy, Part 1: Diagnosis, and neuromuscular, rehabilitation, endocrine, and gastrointestinal and nutritional management. Lancet Neurol. 2018;17:251-67.

5. Ankala A, Kohn JN, Hegde A, Meka A, Ephrem CL, Askree $\mathrm{SH}$, et al. Aberrant firing of replication origins

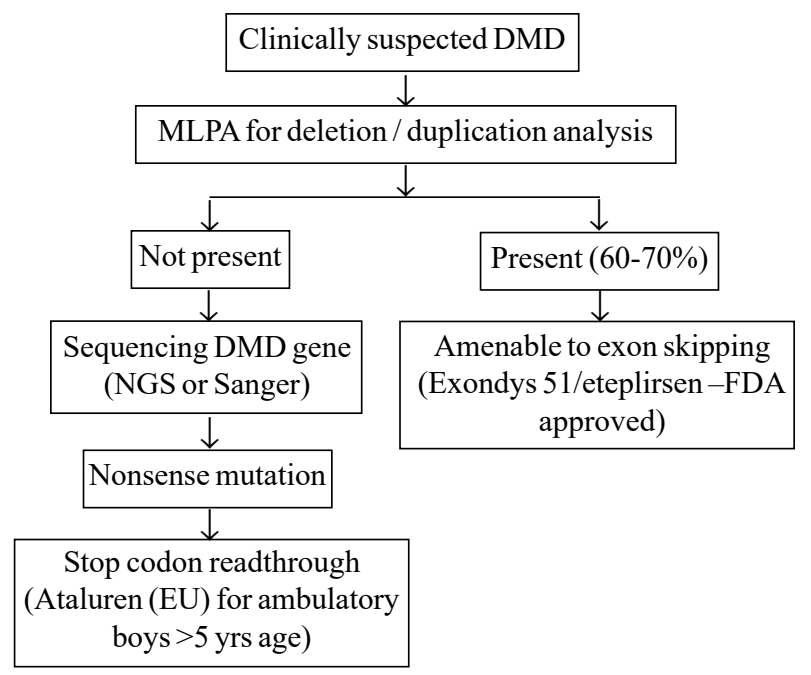

DMD: Duchenne muscular dystrophy; NGS: Next generation sequencing; MLPA: Multiplex ligation dependant probe amplification; FDA: Food and Drugs Administration.

FIG. 1 Precision medicine through molecular diagnosis: DMD as an example

potentially explains intragenic nonrecurrent rearrangements within genes, including the human DMD gene. Genome Res. 2012;22:25-34.

6. Bello L, Pegoraro E. Genetic diagnosis as a tool for personalized treatment of Duchenne muscular dystrophy. Acta Myol. 2016;35:122-27.

7. Aartsma-Rus A, Ginjaar IB, Bushby K. The importance of genetic diagnosis for Duchenne muscular dystrophy. J Med Genet. 2016;53:145-51.

8. McDonald CM, Campbell C, Torricelli RE, Finkel RS, Flanigan KM, Goemans N, et al. Clinical Evaluator Training Group; ACT DMD Study Group. Ataluren in patients with nonsense mutation Duchenne muscular dystrophy (ACT DMD): A multicentre, randomised, double-blind, placebo-controlled, phase 3 trial. Lancet. 2017;390:1489-98. 